\title{
WAVELET-BASED STATISTICAL ANALYSIS FOR OPTICAL IMAGING IN MOUSE OLFACTORY BULB
}

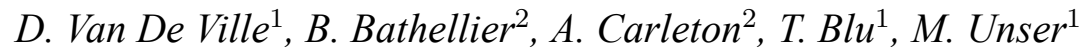 \\ ${ }^{1}$ Biomedical Imaging Group, ${ }^{2}$ Flavor Perception Group (Brain \& Mind Institute) \\ École Polytechnique Fédérale de Lausanne (EPFL) \\ CH-1015 Lausanne, Switzerland
}

\begin{abstract}
Optical imaging is a powerful technique to map brain function in animals. In this study, we consider in vivo optical imaging of the murine olfactory bulb, using an intrinsic signal and a genetically expressed activity reporter fluorescent protein (synaptopHfluorin). The aim is to detect odor-evoked activations that occur in small spherical structures of the olfactory bulb called glomeruli. We propose a new way of analyzing this kind of data that combines a linear model (LM) fitting along the temporal dimension, together with a discrete wavelet transform (DWT) along the spatial dimensions. We show that relevant regressors for the LM are available for both types of optical signals. In addition, the spatial wavelet transform allows us to exploit spatial correlation at different scales, and in particular to extract activation patterns at the expected size of glomeruli. Our framework also provides a statistical significance for every pixel in the activation maps and it has strong type I error control.
\end{abstract}

Index Terms - biomedical optical imaging, olfactory system, wavelet transforms, fluorescence

\section{INTRODUCTION}

In recent years, optical imaging techniques have been successfully deployed to image the functioning of neural networks during sensory stimulation in animals. Two important reasons have contributed to this success. First, these techniques allow to simultaneously observe many points in space, at resolutions ranging from cellular to network scales [1], and time. Second, optical imaging relies on reporter signals that can be linked to activity in various ways; e.g., dye molecules like voltage or calcium sensitives dyes; fluorescent proteins in genetically modified animals; or even an intrinsic signal such as changes in light absorption due to metabolism.

Despite the increasing importance of optical techniques in neuro-imaging, little has been done yet to improve the processing and analysis of optical recordings. Usually, activation maps are obtained by subtraction (or division) of a reference "blank" image. Such an approach clearly does not optimally exploit the full information available in the image time-series; it also lacks the ability to statistically assess the significance of the (relative) intensity changes.

In this paper, we focus on in vivo optical recordings of the olfactory bulb of mice, using an intrinsic signal and a genetically expressed activity reported fluorescent protein (synaptopHfluorin). The goal is to detect odor-evoked activations in the olfactory bulb that are concentrated in small spherical structures named glomeruli.

Recently, we have proposed a framework for the statistical analysis of functional MRI data [2]. This framework forms the basis for the technique that is developed here specifically for the analysis of optical images. The key features of our method are:

1. The temporal information is extracted using the linear model (LM) approach [3]. We show that suitable regressors can be determined for both intrinsic and fluorescence signals.

2. The images are decomposed by means of the spatial discrete wavelet transform (DWT), which provides us with a multi-resolution decomposition of the activation maps. This allows us to select spatial activation patterns at appropriate size and shape (corresponding to the glomeruli activations).

The statistical framework combines the LM and the DWT and provides a sound assessment of the statistical significance of the activation-dependent signal of every pixel. The activation are then detected with a prescribed level of confidence (strong type-I error control). These maps can then be used for further biological interpretation.

\section{WAVELET-BASED STATISTICAL ANALYSIS}

\subsection{Multi-resolution analysis using the DWT}

The DWT [4] decomposes the signal into a weighted sum of basis functions. These are shifted and dilated versions of a (bandpass) wavelet, $\psi(\mathbf{x})$, and shifted versions of a (lowpass) scaling function, $\varphi(\mathbf{x})$. For the ease of notation, we will write 
the 2-D spatial wavelet decomposition of a dataset $v[\mathbf{n}], \mathbf{n} \in$ $\mathbb{Z}^{2}$, as

$$
v[\mathbf{n}]=\sum_{\mathbf{k}} v_{w}[\mathbf{k}] \psi_{\mathbf{k}}(\mathbf{n}),
$$

where $\mathbf{k}$ runs over all subbands and orientations, and $\psi_{\mathbf{k}}$ is the corresponding basis function. In the context of this paper, where we have a series of images, we introduce the temporal dimension as an additional parameter $v[\mathbf{n} ; t], t=1, \ldots, N_{t}$. The practical implementation of the DWT can be done using a fast iterated filterbank algorithm.

\subsection{Temporal modelling using the LM}

We introduce the time-course vector $\mathbf{v}_{w}[\mathbf{k}]=$ $\left[v_{w}[\mathbf{k} ; 1] \ldots v_{w}\left[\mathbf{k} ; N_{t}\right]\right]^{\mathrm{T}}$. The LM explains the temporal behavior of a wavelet coefficient with index $\mathbf{k}$ :

$$
\mathbf{v}_{w}[\mathbf{k}]=\mathbf{X y}_{w}[\mathbf{k}]+\mathbf{e}_{w}[\mathbf{k}]
$$

where the $N_{t} \times L$ design matrix $\mathrm{X}$ contains $L$ regressors, $\mathbf{y}_{w}[\mathbf{k}]$ is the parameter vector of length $L$, and $\mathbf{e}_{w}[\mathbf{k}]$ is the residual error. Under the hypothesis of (temporally) independently and identically gaussian-distributed residuals, the least-squares solution for the parameters is $\overline{\mathbf{y}}_{w}[\mathbf{k}]=$ $\left(\mathbf{X}^{\mathrm{T}} \mathbf{X}\right)^{-1} \mathbf{X}^{\mathrm{T}} \mathbf{v}_{w}[\mathbf{k}]$, and the residual $\overline{\mathbf{e}}_{w}[\mathbf{k}]=\mathbf{v}_{w}[\mathbf{k}]-$ $\mathbf{X} \overline{\mathbf{y}}_{w}[\mathbf{k}]$. The contrast of interest is extracted by a so-called contrast vector $\mathbf{c}$, which results in the measures

$$
\begin{aligned}
u_{w}[\mathbf{k}] & =\mathbf{c}^{\mathrm{T}} \overline{\mathbf{y}}_{w}[\mathbf{k}] \\
s_{w}^{2}[\mathbf{k}] & =\overline{\mathbf{e}}_{w}[\mathbf{k}]^{\mathrm{T}} \overline{\mathbf{e}}_{w}[\mathbf{k}] \mathbf{c}^{\mathrm{T}}\left(\mathbf{X}^{\mathrm{T}} \mathbf{X}\right)^{-1} \mathbf{c} .
\end{aligned}
$$

Note that both the DWT and the LM are linear operations. Consequently, their order of execution can be interchanged and $u_{w}[\mathbf{k}]$ would correspond to the DWT of the contrast map. However, in the next part, we introduce a powerful non-linear treatment in the wavelet domain.

\subsection{Framework for wavelet-based statistical analysis}

\subsubsection{Adaptive denoising}

The wavelet coefficients $u_{w}[\mathbf{k}]$ are denoised in the wavelet domain based on both their estimated value and their residual error. For that purpose, we consider the proportion

$$
t_{w}[\mathbf{k}]=\frac{u_{w}[\mathbf{k}]}{\sqrt{s_{w}^{2}[\mathbf{k}] / J}}, \text { with } J=N_{t}-\operatorname{rank}(\mathbf{X}),
$$

which should follow a Student $t$-distribution in the absence of contrast (i.e., no response). The reconstruction after hard thresholding is

$\tilde{u}[\mathbf{n}]=\sum_{\mathbf{k}} \tilde{u}_{w}[\mathbf{k}] \psi_{\mathbf{k}}(\mathbf{n})$, where $\begin{cases}\tilde{u}_{w}[\mathbf{k}]=u_{w}[\mathbf{k}], & \text { when }\left|t_{w}[\mathbf{k}]\right|>\tau_{w} \\ 0, & \text { otherwise }\end{cases}$

where $\tau_{w}$ is a threshold parameter. Notice that we explicitly did not introduce the notion of a statistical test here.

\subsubsection{Statistical detection}

Our aim is to establish the statistical significance of the contrast in the spatial domain in order to test the hypotheses

$$
\mathcal{H}_{0}: E[\tilde{u}[\mathbf{n}]]=0, \quad \mathcal{H}_{1}: E[\tilde{u}[\mathbf{n}]]>0 .
$$

As a result of the theorem in [2], the test procedure relies on the null hypothesis rejection inequality:

$$
\operatorname{Prob}\left[\frac{\tilde{u}[\mathbf{n}]}{\Lambda[\mathbf{n}]} \geq \tau_{s}\right] \leq \Upsilon\left(\tau_{w}, \tau_{s}\right)
$$

where $\Upsilon\left(\tau_{w}, \tau_{s}\right)$ is data-independent, and $\Lambda[\mathbf{n}]$ is a special reconstruction of the residuals:

$$
\Lambda[\mathbf{n}]=\sum_{\mathbf{k}} \frac{s_{w}[\mathbf{k}]}{\sqrt{J}}\left|\psi_{\mathbf{k}}(\mathbf{n})\right| .
$$

In practice, the threshold values $\tau_{w}$ and $\tau_{s}$ are obtained by fixing $\Upsilon\left(\tau_{w}, \tau_{s}\right)=\alpha / N$, where $\alpha$ is the desired global significance level and $N$ the number of pixels.

\subsection{Summary of the method}

1. Apply the spatial DWT to the measured data $v[\mathbf{n} ; t]$ to obtain the wavelet coefficients $v_{w}[\mathbf{k} ; t]$.

2. Apply the LM to the temporal dimension and extract the "contrast" of interest $u_{w}[\mathbf{k}]$ with its residual error $s_{w}^{2}[\mathbf{k}]$.

3. Apply the wavelet denoising step to obtain $\tilde{u}_{w}[\mathbf{k}]$.

4. Apply the inverse DWT to $\tilde{u}_{w}[\mathbf{k}]$ and the "absolute value" inverse DWT to $s_{w}[\mathbf{k}]$. We obtain $\tilde{u}[\mathbf{n}]$ and $\Lambda[\mathbf{n}]$.

5. Apply the detection step $\tilde{u}[\mathbf{n}] / \Lambda[\mathbf{n}]>\tau_{s}$.

\section{EXPERIMENTAL SETUP}

The experimental setup is shown in Fig. 1. We briefly highlight the most important specifications.

\subsection{Intrinsic signal acquisition}

The olfactory bulb is illuminated by red light $(700 \mathrm{~nm} ; 100 \mathrm{~W}$ halogen lamp). The images are measured through an objective (total magnification 7.9) by a CCD camera (Imager 3001F, Optical Imaging, Mountainside NJ; resolution $180 \times$ 252 after binning; $5 \mathrm{~Hz}$ frame rate).

\subsection{Fluorescence signal acquisition}

The olfactory bulb is illuminated with 480nm light (filter HQ 480/40), and reflected through a dichroic mirror (Q505LP). Emitted light then passes through a $535 \mathrm{~nm}$ band-pass filter (HQ535/50). Images are captured using a CMOS camera (Micam Ultima, Brainvision, Tokyo, Japan; resolution $100 \times 100 ; 25 \mathrm{~Hz}$ frame rate). 


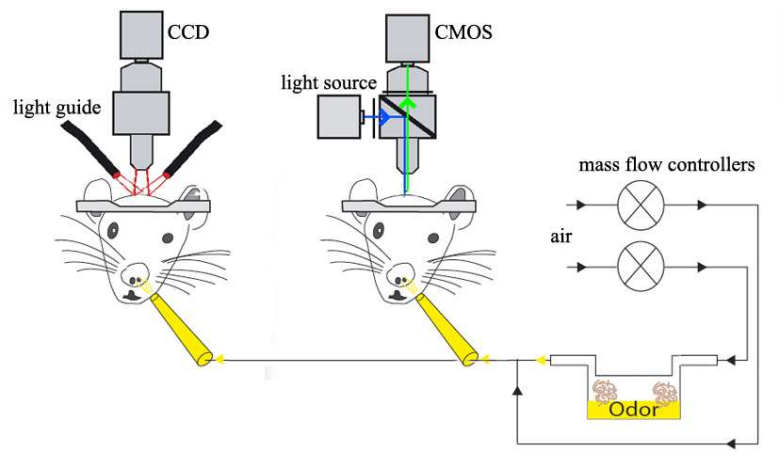

Fig. 1. Sketch of the optical imaging setup and odor stimulation apparatus for imaging the intrinsic signal (left) and the fluorescence signal (right).

\subsection{Odor stimulation}

Odors are delivered for a $5 \mathrm{~s}$ period by an olfactometer. The air flow is purified with a charcoal filter and then split into a dilution stream and an odor stream, which are merged again before the output. Before exposing the mouse to the odor, steady state is assured by directing during $5 \mathrm{~s}$ to an exhaust circuit. Further details on the setup can be found in [5].

\section{REGRESSORS FOR OPTICAL IMAGING}

\subsection{Intrinsic signal}

Observation of intrinsic signals [6] suggests an exponentially decaying signal after stimulation. Therefore, we propose the following model to explain this signal:

$$
f(t)=y_{1} \cdot\left(e^{-\frac{t-t_{0}}{\tau_{\mathrm{ac}}}}-1\right) H_{t-t_{0}}+y_{2}+e(t),
$$

where $t_{0}$ is the (known) stimulus onset, $\tau_{\text {ac }}$ is the time constant of the decaying exponential, $H_{t}$ is the step function, $e(t)$ is the noise term, and $y_{1}, y_{2}$ are the linear weights representing the activation-dependent intensity and constant background, respectively. To be suitable for a LM approach, the regressors need to be fixed and only the linear weights are left to be estimated. We fixed the time constants by a nonlinear fit of (9) for a pool of 510 presentations (10 animals). We obtained as an average value (with standard deviation): $\tau_{\mathrm{ac}}=2.18 \pm 1.36 \mathrm{~s}$.

\subsection{Fluorescence signal}

Observation of fluorescence signals reveals a more complex structure. Next to an activation-dependent component similar to the intrinsic signal, there is an important influence of photo-bleaching (resulting in a consistent decay), as well as a

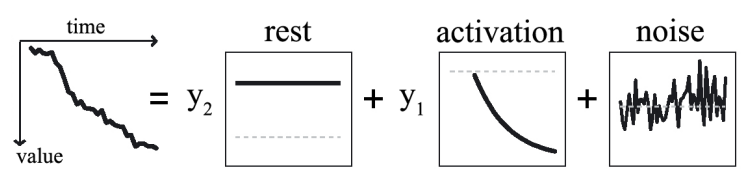

(a)

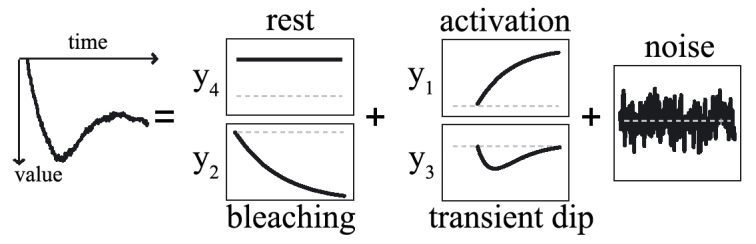

(b)

Fig. 2. Schematic overview of the regressors included in the LM to explain (a) the intrinsic signal, (b) the fluorescence signal.

transient dip. We propose the following model:

$$
\begin{aligned}
f(t)= & y_{1} \cdot\left(1-e^{-\frac{t-t_{0}}{\tau_{\mathrm{ac}}}}\right) H_{t-t_{0}}+y_{2} \cdot\left(1-e^{-\frac{t}{\tau_{\mathrm{b}}}}\right)+ \\
& y_{3} \cdot\left(e^{-\frac{t-t_{0}}{\tau_{\mathrm{d}}}}-e^{-\frac{t-t_{0}}{\tau_{\mathrm{r}}}}\right) H_{t-t_{0}}+y_{4}+e(t),(10)
\end{aligned}
$$

where the linear weigths $y_{1}, y_{2}, y_{3}, y_{4}$ represent the intensities of the activation, bleaching, transient dip, and background signal, respectively. The non-linear parameters are again estimated by a non-linear fit for a pool of 100 presentations (5 animals). We obtained the values: $\tau_{\mathrm{ac}}=1.15 \pm 0.6 \mathrm{~s}$, $\tau_{\mathrm{r}}=0.96 \pm 0.4 \mathrm{~s}, \tau_{\mathrm{d}}=1.26 \pm 0.4 \mathrm{~s}$.

\section{GLOMERULI SELECTION}

For the analysis of image time-series from the olfactory bulb, the multi-resolution decomposition provided by the DWT can be exploited most advantageously. It is easy to see that the null rejection inequality is still valid when we replace $\tilde{u}[\mathbf{n}]$ by

$$
\min \left(\tilde{u}[\mathbf{n}], \sum_{\mathbf{k} \in \mathcal{S}} \tilde{u}_{w}[\mathbf{k}] \psi_{\mathbf{k}}(\mathbf{n})\right),
$$

where $\mathcal{S}$ is a set of indices corresponding to a selection of wavelet coefficients (e.g., subbands).

For B-spline wavelets (degree $n$ ), we can determine the characteristic size of features that will be captured at a certain scale. Following [7,8], we find the diameter (full width at half maximum) of the equivalent Gaussian in a low-pass after $J_{w}$ iterations:

$$
D_{\text {eq }}\left(J_{w}\right)=\sqrt{2 \ln (2)} \sqrt{n+1} \sqrt{\frac{4^{J_{w}}-1}{3}} .
$$

For the orthogonal cubic B-spline DWT ( $n=3, J_{w}=6$ iterations) and a resolution of $12.5 \mu \mathrm{m} / \mathrm{pixel}$, we obtain equivalent sizes of $30,66,135,271,544$, and $1088 \mu \mathrm{m}$. 


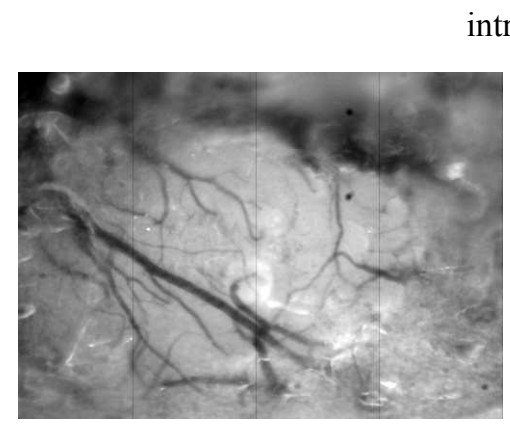

(a)

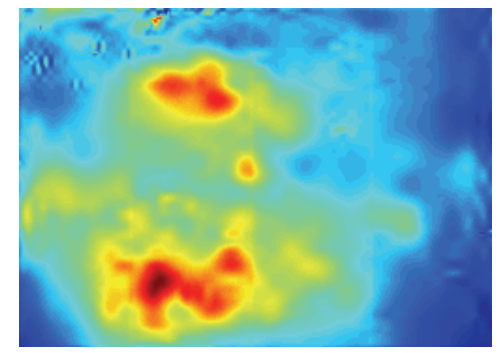

(c) intrinsic signal

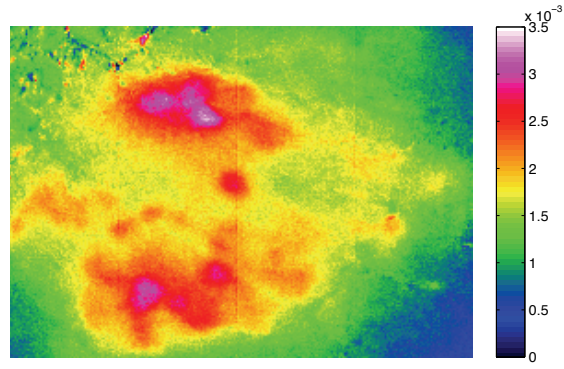

(b)

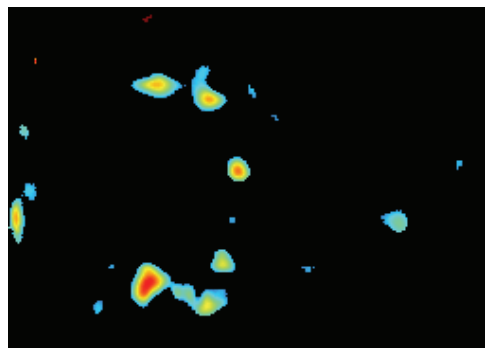

(d) fluorescence signal

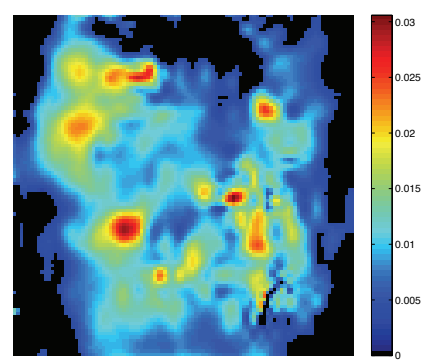

(e)

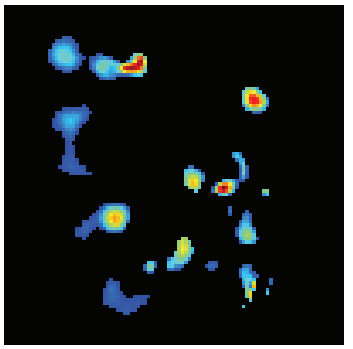

(f)

Fig. 3. Experimental results. Intrinsic signal for methyl benzoate $(2 \times$ diluted): (a) Blood vessel image acquired with green light (546nm); (b) Parameter map for activation-dependent regressor without any wavelet denoising or statistical testing; (c) Parameter map after wavelet denoising and statistical testing (all pixels are significant); (d) Parameter map after wavelet denoising and statistical testing but with subband selection to retain glomeruli-like structures only. Fluorescence signal for ethyl butyrate (50× diluted): (e) Parameter map after wavelet denoising and statistical testing; (f) Same as (e) but with subband selection.

\section{RESULTS}

The significance level is fixed at $\alpha=0.1 \%$, which allows us to have high confidence in the detected activations since the probability of a type I error (false positive) is controlled at this level. The corresponding threshold values are $\tau_{w}=7.64$ and $\tau_{s}=0.30$.

Two examples are shown in Fig. 3. The glomeruli selection retains all subbands for features below $70 \mu \mathrm{m}$. The maps (d) and (f) are useful for further biological interpretation.

We also compared our method against spatial band-pass filtering and manual selection, for which we refer to [5].

\section{CONCLUSION}

We propose to extend the wavelet-based statistical framework for optical imaging. In particular, we showed its suitability for processing intrinsic and fluorescence signals from mouse olfactory bulb.

\section{ACKNOWLEDGEMENTS}

This was supported by the Brain Mind Institute (EPFL) and the Center for Biomedical Imaging (CIBM) of the Geneva-Lausanne Universities and the EPFL, as well as the foundations Leenaards and Louis-Jeantet.

\section{REFERENCES}

[1] A. Grinvald and R. Hildesheim, "VSDI: a new era in functional imaging of cortical dynamics," Nat. Rev. Neurosci., vol. 5, pp. 874-885, 2004.

[2] D. Van De Ville, T. Blu, and M. Unser, "Integrated wavelet processing and spatial statistical testing of fMRI data," NeuroImage, vol. 23, no. 4, pp. 1472-1485, Dec. 2004.

[3] G. A. F. Seber and A. J. Lee, Linear Regression Analysis, John Wiley \& Sons, 2003.

[4] S. Mallat, "A theory for multiresolution signal decomposition: The wavelet decomposition," IEEE Trans. Pattern Anal. Mach. Intell., vol. 11, pp. 674-693, 1989.

[5] B. Bathellier, D. Van De Ville, T. Blu, M. Unser, and A. Carleton, "Wavelet-based multi-resolution statistics for optical imaging signals: application to automated detection of odour activated glomeruli in the mouse olfactory bulb," NeuroImage, vol. 34, pp. 1020-1035, 2007.

[6] M. Meister and T. Bonhoeffer, "Tuning and topography in an odor map on the rat olfactory bulb," J. Neurosci., vol. 21, pp. 1351-1360, 2001.

[7] D. Van De Ville, T. Blu, and M. Unser, "Wavelets versus resels in the context of fMRI: establishing the link with SPM," in SPIE's Symposium on Optical Science and Technology: Wavelets X, San Diego CA, USA, Aug. 2003, SPIE, vol. 5207.

[8] M. J. Fadili and E. T. Bullmore, "A comparative evaluation of wavelet-based methods for multiple hypothesis testing of brain activation maps," NeuroImage, vol. 23, no. 3, pp. 1112-1128, 2004. 